\title{
Fabrication of Invisible Ag Nanowire Electrode Patterns Based on Laser-Induced Rayleigh Instability
}

Harim Oh, Jeeyoung Lee, Jin-Hoon Lim, Jin-Woo Park and Myeongkyu Lee*

Department of Materials Science and Engineering, Yonsei University, Seoul 120-749, Korea

SUPPORTING INFORMATION

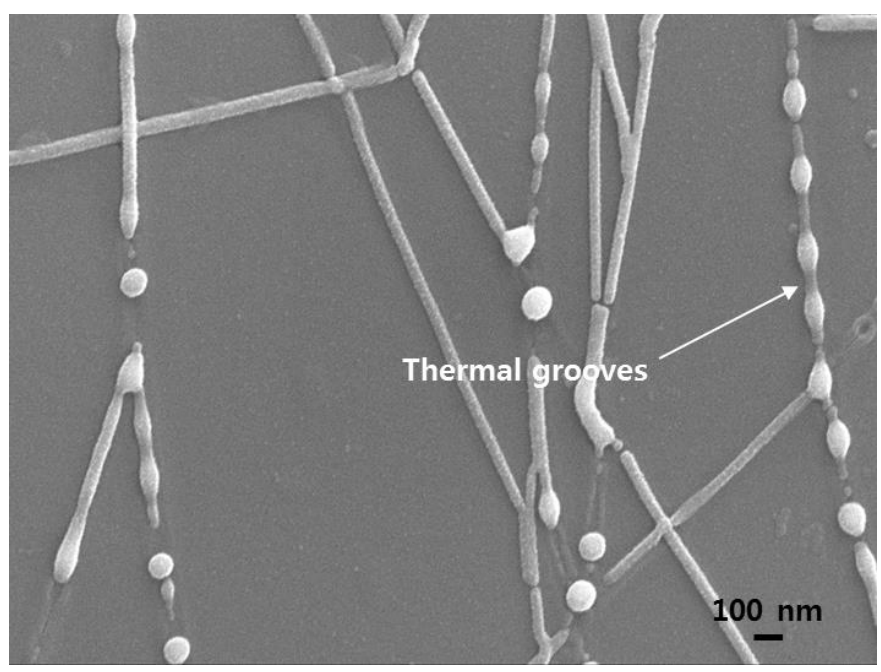

Figure S1 SEM image showing the decomposition of Ag nanowires into smaller droplets 

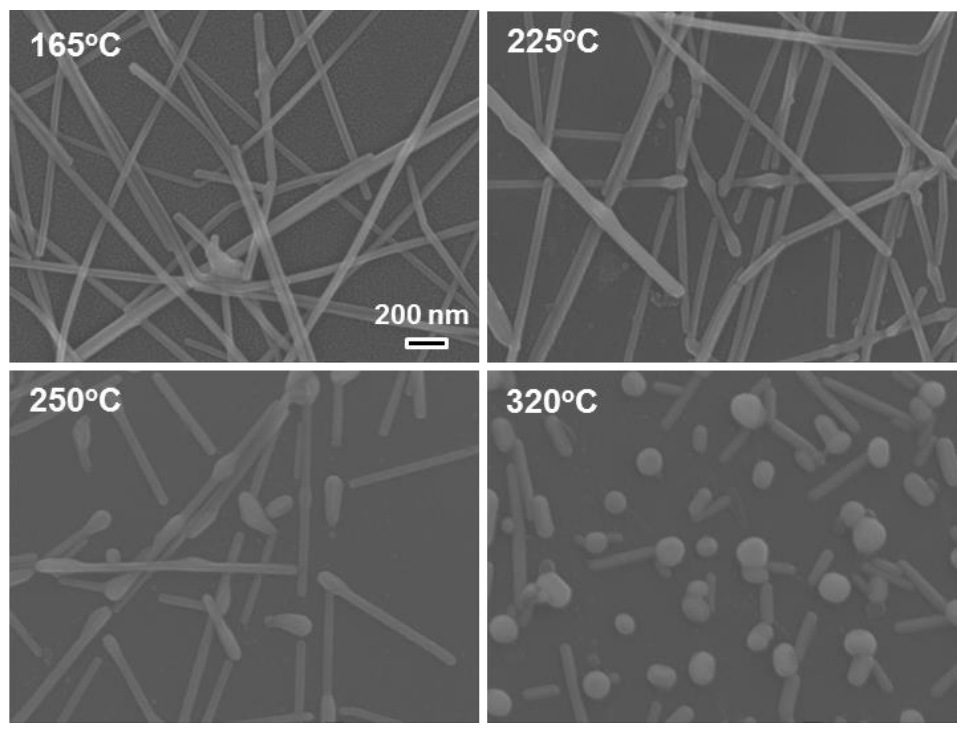

Figure S2 Morphology change of AgNWs as a function of heat treatment temperature. AgNW films coated onto glass substrates were heat-treated in ambient air.

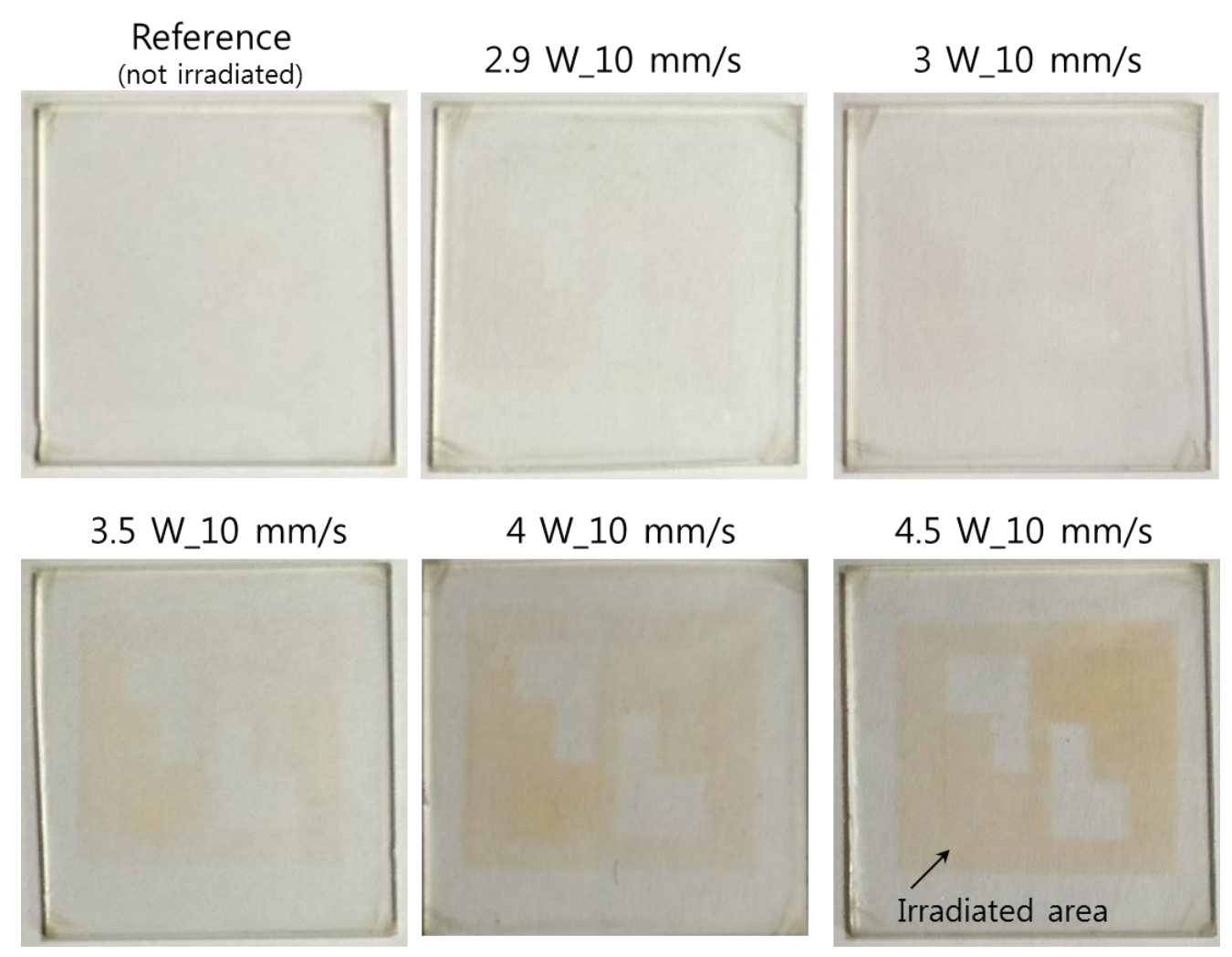

Figure S3 Visibility of the color caused by the Ag nanoparticles. AgNW films were coated using a $0.3 \mathrm{wt} \%$ solution in ethanol. The sample sizes are $2 \mathrm{~cm} \times 2 \mathrm{~cm}$. 
Table S1 Sheet resistance vs. laser power as measured with multiple samples (unit: $\Omega / \mathrm{sq}$ )

\begin{tabular}{|c|c|c|c|c|c|c|c|}
\hline Samples & Reference & $2.8 \mathrm{~W}$ & $2.9 \mathrm{~W}$ & $3.0 \mathrm{~W}$ & $3.5 \mathrm{~W}$ & $4.0 \mathrm{~W}$ & $4.5 \mathrm{~W}$ \\
\hline$\# 1$ & 29 & 670 & 3855 & 860 & $\infty$ & $\infty$ & $\infty$ \\
\hline$\# 2$ & 28 & $\infty$ & $\infty$ & $\infty$ & $\infty$ & $\infty$ & $\infty$ \\
\hline$\# 3$ & 31 & 335 & 786 & 1421 & $\infty$ & $\infty$ & $\infty$ \\
\hline$\# 4$ & 32 & $\infty$ & $\infty$ & $\infty$ & $\infty$ & $\infty$ & $\infty$ \\
\hline$\# 5$ & 32 & 405 & $\infty$ & $\infty$ & $\infty$ & $\infty$ & $\infty$ \\
\hline$\# 6$ & 28 & 257 & $\infty$ & $\infty$ & $\infty$ & $\infty$ & $\infty$ \\
\hline$\# 7$ & 34 & $\infty$ & $\infty$ & $\infty$ & $\infty$ & $\infty$ & $\infty$ \\
\hline$\# 8$ & 28 & 326 & 1619 & $\infty$ & $\infty$ & $\infty$ & $\infty$ \\
\hline
\end{tabular}

